\title{
Analysis of period doubling bifurcation and chaos mirror of biped passive dynamic robot gait
}

\author{
ZHAO Jie, WU XiaoGuang ${ }^{*}$, ZANG XiZhe \& YAN JiHong \\ State Key Laboratory of Robotics and System, Harbin Institute of Technology, Harbin 150080, China
}

Received November 16, 2011; accepted February 3, 2012

\begin{abstract}
With a reasonable parameter configuration, the passive dynamic walking model has a stable, efficient, natural periodic gait, which depends only on gravity and inertia when walking down a slight slope. In fact, there is a delicate balance in the energy conversion in the stable periodic gait, making the gait adjustable by changing the model parameters. Poincaré mapping is combined with Newton-Raphson iteration to obtain the numerical solution of the final state of the passive dynamic walking model. In addition, a simulation on the walking gait of the model is performed by increasing the slope step by step, thereby fixing the model's parameters synchronously. Then, the gait features obtained in the different slope stages are analyzed and discussed, the intrinsic laws are revealed in depth. The results indicate that the gait can present features of a single period, doubling period, the entrance of chaos, merging of sub-bands, and so on, because of the high sensitivity of the passive dynamic walking to the slope. From a global viewpoint, the gait becomes chaotic by way of period doubling bifurcation, with a self-similar Feigenbaum fractal structure in the process. At the entrance of chaos, the gait sequence comprises a Cantor set, and during the chaotic stage, sub-bands in the final-state diagram of the robot system present as a mirror of the period doubling bifurcation.
\end{abstract}

period doubling bifurcation, chaos, fractals, passive dynamic walking, final-state diagram, Feigenbaum constant

Citation: $\quad$ Zhao J, Wu X G, Zang X Z, et al. Analysis of period doubling bifurcation and chaos mirror of biped passive dynamic robot gait. Chin Sci Bull, 2012, 57: 1743-1750, doi: 10.1007/s11434-012-5113-3

In the past decades, there has been great progress in biped walking robots, with many types of walking robots having been developed. Vast energy consumption and complex control, however, presented challenges until McGeer's proposal of passive dynamic walking [1].

A biped passive dynamic robot takes good advantage of its gravity and inertia in the walking process instead of control in terms of gait features. Thus, it has two special inherent properties: stability and low energy consumption [2-7]. However, these special properties lead to high sensitivity of the gait stability to the inner structural parameters and external disturbances; any tiny change in parameters will cause an obvious change in the gait $[8,9]$.

The ultimate goal of the biped passive dynamic walking robot is to realize natural and efficient humanoid walking under different conditions. In most cases, stable walking of

*Corresponding author (email: wxgtreera@gmail.com) human beings can be considered as motion with a cycle of one step $[10,11]$. However, humans are able to achieve aperiodic gait by controlling the muscle parameters, such as changing the step length or step speed of any walking gait [12]. If the structural parameters or the slope changes, the passive dynamic walking robot will have doubling-periodic gait or aperiodic gait besides the single-period motion. This is performed by the auto-adjustment of the gait according to the balance of energy conversion.

Liu et al. [13] and Asano et al. [14,15] researched the effect of the main parameters of the straight leg compass-gait model on single-period stable gait. As the slope gradually increases, the passive dynamic walking robot displays periodic gait and chaotic gait successively, as represented in the simplest model [16], compass-gait model [17], kneed model [18], and upper body model [19]. Furthermore, the study in [17] identified period-doubling bifurcation gait, which eventually leads to chaos, while that in [18] revealed that the 
ratios of the distances between successive bifurcation values on the slope axis are expected to converge to the Feigenbaum constant. Liu et al. [20] adopted Newton-Raphson iteration to analyze the change rule of eigenvalues of the Jacobi Matrix when period bifurcation occurs as the slope increases. In addition, some researchers restrained the period bifurcation and chaos caused by an increase in the slope through the conservation of energy [21-24].

Meanwhile, various different types of chaotic phenomena in other fields have been investigated [25-27].

Thus far, however, there has been no research on the way that a robot enters the chaotic state, the dynamic features at the entrance to chaos, or the relationship between the period doubling mirror and the delicate structural branches in the sub-band abruption.

Moreover, the effect of the parameters on the way that a robot enters the chaotic state has also not been researched in the present days. In particular, two aspects need to be investigated, that is, an analysis of the dynamic features at the entrance to chaos and the relationship between the period doubling mirror and the delicate structural branches in the sub-band abruption. Nevertheless, both the robot's structural parameters and a slope change can lead to a chaotic gait. The former is maintained in the motion process, which consequently can be considered before the design of the model to remove the periodic gait and aperiodic gait resulting from it. Thus, only the effect of the slope on the chaotic gait is discussed in this paper. Some methods in the analysis refer to the quadratic system presented in [25]. This study aims to reveal intrinsic laws of the period doubling bifurcation and chaos by researching the effect the slope has on the walking gait of a biped passive dynamic walking robot. This provides a theoretical foundation for the restraint and control strategies for periodic and chaotic gaits.

\section{Model}

The robot studied in this paper is a 2-D walker with 2 straight legs, each of which comprises 2 leg-structures. Two medial and two lateral leg-structures are also connected, thus solving the problem of lateral instability. In fact, the robot is a biped walking robot model, composed of two rigid straight legs connected by a passive hinge. A sketch of the model is shown in Figure 1. All movements are limited in the plane depicted in Figure 1. The two straight legs in the model have the same mass and geometric parameters. Both legs are homogeneous. As shown in the figure, the mass of each leg is denoted by $m$, the moment of inertia relative to the centre of mass of each leg by $J_{1}$, the length by $l$, and the distance between the centre of the mass and the mass point of the hip by $c$. The mass of the hip is denoted by $m^{\prime}$. To make the motivation more stable, the robot has arced feet, with a radius of $r$. The model can be considered to be a straight-leg compass-gait model, because the movement of

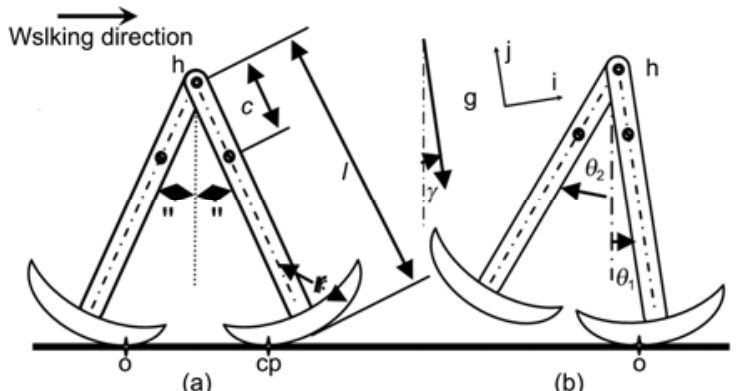

Figure 1 Sketch of the model (a) collision stage; (b) swing stage.

each leg is regarded as a double pendulum motion.

To reduce the number of parameters and make the dynamics equation more universal, the parameters are made non-dimensional by setting $r=r / l, c=c / l, m^{\prime}=m^{\prime} / m, J_{1}=J_{1} / m l^{2}$, and rescaling the time as $t=t \sqrt{(g / l)}$.

Meanwhile, the following assumptions are made: (1) legs are rigid without elastic deformation, and the hip is free of damping or friction; (2) the contact between the foot and the floor is idealized since rounded feet cannot deform themselves or slip; the impact is modeled as an instantaneous and fully inelastic impact where nether slip nor bounce occurs; and (3) the robot is assumed to walk on a rigid, flat and shallow slope.

In this paper, the effect of the slope $\gamma$ on the walking gait, i.e., the changing trend in the state variables of the stable gait as $\gamma$ increases, is analyzed in detail. Let the main parameters of the robot model be fixed as: $c=0.15, m^{\prime}=2$, $J_{1}=0.01, r=0.3$.

\section{Dynamic modeling}

Under gravity, inertia and reasonable initial conditions, the passive dynamic model is simulated to walk down a shallow slope stably. This motion process is divided into the following two stages.

\subsection{The swing stage}

Once the swing leg leaves the slope, the stance leg performs the inverted pendulum motion. Then the swing leg performs a single pendulum motion around the fulcrum that moves along the arc trajectory. In this stage, only gravity is at work, so mechanical energy is conserved during this stage. The dynamic equations of motion are derived from the Lagrange equation in the following form:

$$
\left[\begin{array}{ll}
M f_{11} & M f_{12} \\
M f_{21} & M f_{22}
\end{array}\right]\left[\begin{array}{l}
\ddot{\boldsymbol{\theta}}_{1} \\
\ddot{\boldsymbol{\theta}}_{2}
\end{array}\right]=\left[\begin{array}{l}
F f_{1}\left(\boldsymbol{\theta}_{1}, \dot{\boldsymbol{\theta}}_{1}, \boldsymbol{\theta}_{2}, \dot{\boldsymbol{\theta}}_{2}\right) \\
F f_{2}\left(\boldsymbol{\theta}_{1}, \dot{\boldsymbol{\theta}}_{1}, \boldsymbol{\theta}_{2}, \dot{\boldsymbol{\theta}}_{2}\right)
\end{array}\right] .
$$

\subsection{The collision stage}

We have adopted three idealized assumptions in building 
the dynamic equations for this stage: (1) the collision between the swing leg and the slope is instantaneous, at which time the roles of the swing leg and stance leg are exchanged; (2) since the only external force occurs at the point of impact, there are no moments created around this point; and (3) the angular momentum is then conserved for the entire system about the collision point and for the swing leg after the impact about the hip. Let "-" and "+" denote, respectively, the state before and after the collision. For convenience of notation, we use h to denote the hip of the robot, st, sw to denote the stance leg and swing leg, respectively, stc for the center of the mass of the stance leg, swc for the center of the mass of the swing leg, $\boldsymbol{r}_{s t c / c p}^{-}$for the vector from the contact point to stc before the collision, $\boldsymbol{r}_{s w c / c p}^{-}$for the vector from the contact point to swc before the collision, $\boldsymbol{V}_{h}^{-}$for the speed of $\mathrm{h}$ before the collision, $\boldsymbol{V}_{s t c}^{-}$for the speed of stc before the collision, and $\boldsymbol{V}_{s w c}^{-}$for the speed of swc before the collision. $\boldsymbol{k}$ is a vector of size $\boldsymbol{i} \times \boldsymbol{j}$, with $\boldsymbol{i}, \boldsymbol{j}$ as shown in Figure 1. Vectors of $\boldsymbol{r}$ and $\boldsymbol{V}$ annotated with the symbol "+" occur after the collision.

From the conservation of angular momentum of the whole robot system about the contact point $\mathrm{cp}$, we get

$$
\begin{aligned}
& \boldsymbol{r}_{s t c / c p}^{-} \times \boldsymbol{V}_{s t c}^{-}+J_{1} \dot{\boldsymbol{\theta}}_{1}^{-} \boldsymbol{k}+\boldsymbol{r}_{s w c / c p}^{-} \times \boldsymbol{V}_{s w c}^{-}-J_{1} \dot{\boldsymbol{\theta}}_{2}^{-} \boldsymbol{k}+\boldsymbol{r}_{h / c p}^{-} \times m^{\prime} \boldsymbol{V}_{h}^{-} \\
& =\boldsymbol{r}_{s t c / c p}^{+} \times \boldsymbol{V}_{s t c}^{+}+J_{1} \dot{\boldsymbol{\theta}}_{1}^{+} \boldsymbol{k}+\boldsymbol{r}_{s w c / c p}^{+} \times \boldsymbol{V}_{s w c}^{+}-J_{1} \dot{\boldsymbol{\theta}}_{2}^{+} \boldsymbol{k}+\boldsymbol{r}_{h / c p}^{+} \times m^{\prime} \boldsymbol{V}_{h}^{+}
\end{aligned}
$$

And, from the conservation of angular momentum of the swing leg after the collision about the hip $\mathrm{h}$, we get

$$
\boldsymbol{r}_{s t c / h}^{-} \times \boldsymbol{V}_{s t c}^{-}-J_{1} \dot{\boldsymbol{\theta}}_{1}^{-} \boldsymbol{k}=\boldsymbol{r}_{s w c / h}^{+} \times \boldsymbol{V}_{s w c}^{+}+J_{1} \dot{\boldsymbol{\theta}}_{2}^{+} \boldsymbol{k} .
$$

Simplifying eqs. (2) and (3), we have

$$
\left[\begin{array}{cc}
A f_{11} & A f_{12} \\
A f_{21} & A f_{22}
\end{array}\right]\left[\begin{array}{c}
\dot{\boldsymbol{\theta}}_{1}^{-} \\
\dot{\boldsymbol{\theta}}_{2}^{-}
\end{array}\right]=\left[\begin{array}{ll}
B f_{11} & B f_{12} \\
B f_{21} & B f_{22}
\end{array}\right]\left[\begin{array}{c}
\dot{\boldsymbol{\theta}}_{1}^{+} \\
\dot{\boldsymbol{\theta}}_{2}^{+}
\end{array}\right] .
$$

As eq. (4) shows, the stance and swing legs are exchanged at the time of impact, so $\boldsymbol{\theta}_{1}^{+}=\boldsymbol{\theta}_{2}^{-}$and $\boldsymbol{\theta}_{2}^{+}=\boldsymbol{\theta}_{1}^{-}$.

\subsection{Solution of the model}

The dynamic model of a biped passive dynamic walking robot is a non-linear continuous dynamic system, the analytical and numerical computation of which is quite difficult. Poincaré proposed a method, called Poincaré mapping, to transform a non-linear continuous dynamic system into a discrete dynamic system preserving the main features. For the passive dynamic walking robot in this work, we use a state vector at one time of the walking period to represent the dynamic features of the whole walking period.

In general, the space in which the state vector exists after the collision, is referred to as the Poincaré section. Meanwhile, the swing leg has the geometric relationship $\boldsymbol{\theta}_{1}^{+}=$ $-\boldsymbol{\theta}_{2}^{+}$with the stance leg. Thus, the independent state variables are $\boldsymbol{\theta}_{1}, \dot{\boldsymbol{\theta}}_{1}$, and $\dot{\boldsymbol{\theta}}_{2}$, comprising the state vector $\boldsymbol{v}=\left[\boldsymbol{\theta}_{1}, \dot{\boldsymbol{\theta}}_{1}, \dot{\boldsymbol{\theta}}_{2}\right]^{\mathrm{T}}$. The dynamic features of the system can be described by the transform $S$ defined on the section. The definition of $S$ is given in eq. (5), where $\boldsymbol{v}_{n}$ is the value of the state vector $v$ in the $n$-th step of walking in the Poincare section, and Poincaré mapping calculates the next state vector $\boldsymbol{v}_{n+1}$ according to the current state vector $\boldsymbol{v}_{n}$.

$$
\boldsymbol{v}_{n+1}=S\left(\boldsymbol{v}_{n}\right) .
$$

Because of the high nonlinearity of the system, the analytical form of the Poincaré mapping $S$ is difficult to determine; the Newton-Raphson numerical method is adopted. If there is fixed point $v^{*}$ in the system, then it must obey: $v^{*}=S\left(v^{*}\right)$

\section{Period doubling chaos mirror}

\subsection{Period doubling phenomenon}

This is explained by the following model solution. The Poincaré section in the phase space orbit can simplify the complicated motion, so geometric intuition methods on the Poincaré section are used to describe all states in the dynamic system of the biped robot. For a given value of $\gamma$, beginning with a reasonable initial value, after several iterative transition states, the orbit converges to a fixed points sequence, which is called the final state. Figure 2 shows the final-state diagram of $\boldsymbol{\theta}_{1}$ on the Poincaré section with $\gamma \in[0.13,0.172677]$. From the view of the bifurcation, the gait cycle is continually divided from one cycle into two: when period 1 is unstable, it bifurcates period 2 ; when period 2 is unstable, it bifurcates period $4 ; \cdots$; when period $2^{n-1}$ is unstable, it bifurcates period $2^{n}$. This process is referred to as period doubling bifurcation.

(i) The period doubling bifurcation series. The parameter sequence at the bifurcation points $\gamma_{1}, \gamma_{2}, \gamma_{3} \cdots$ is shown in Figure 3 , where $\gamma_{1}=0.139622$ denotes the bifurcation value from 1 to $2, \gamma_{2}=0.1596231$ denotes the bifurcation value

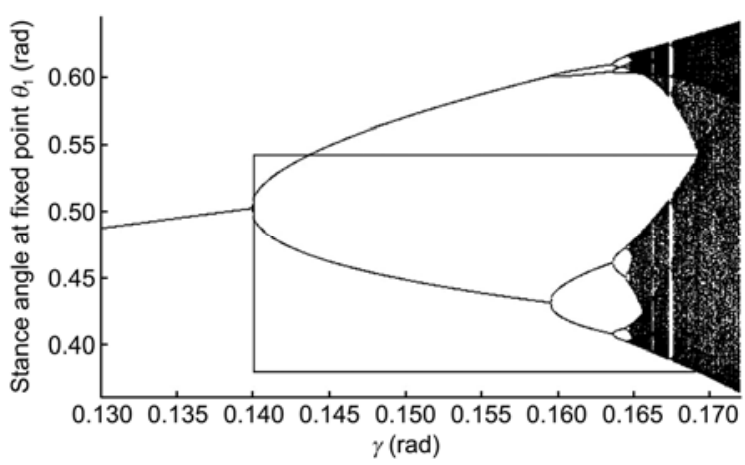

Figure 2 The final-state diagram of $\boldsymbol{\theta}_{1}$ on the Poincaré section. 


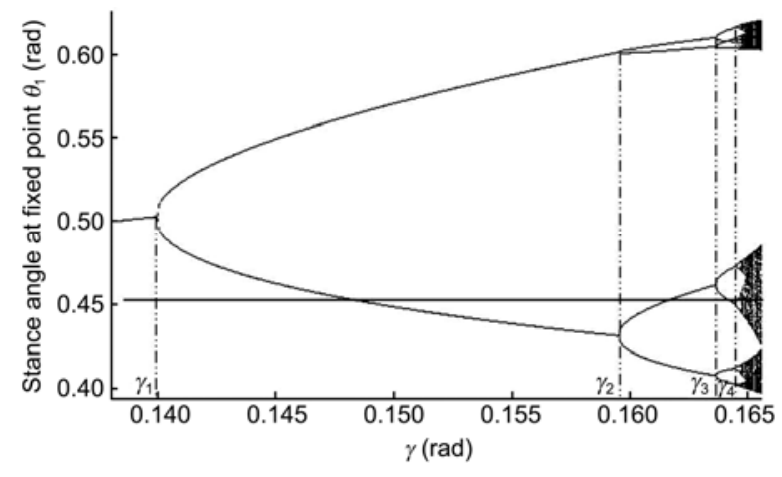

Figure 3 Each level of bifurcation points in the period doubling bifurcation.

from 2 to 4 , and so on. Then, the ratio of the difference in $\gamma$ between 2 adjacent bifurcation values is calculated: $\left(\gamma_{n}-\gamma_{n-1}\right) /$ $\left(\gamma_{n+1}-\gamma_{n}\right), n=2,3, \cdots$, giving the results shown in Table 1.

There is a period area in the final-state diagram with $\gamma \in(0.1351,0.1647)$. In this area, there is a positive $2^{n}(n=$ $0,1,2, \cdots)$ period doubling bifurcation sequence. As seen in Table 1, there is a limit to the ratio of differences in $\gamma$ between 2 adjacent bifurcation points.

$$
\delta=\lim _{n \rightarrow \infty} \frac{\left(\gamma_{n}-\gamma_{n-1}\right)}{\left(\gamma_{n+1}-\gamma_{n}\right)} .
$$

(ii) The universal constant. Between adjacent bifurcation points, distance reduction in the horizontal direction does not follow as a geometric progression. As $\gamma$ increases, it merely approximates a geometric progression by the ratio of $\delta_{n}=\left(\gamma_{n}-\gamma_{n-1}\right) /\left(\gamma_{n+1}-\gamma_{n}\right)$, which converges as $n$ increases. Feigenbaum calculated the following:

$$
\lim _{n \rightarrow \infty} \delta_{n}=\delta=4.66920160910299 \cdots,
$$

where $\delta$ is an irrational number, called the Feigenbaum constant. The occurrence of this constant in many different systems is referred to as the universality and expresses that these types of systems have the same bifurcation speed when approaching chaos from period doubling bifurcation. Thus, we can predict what the parameter is when the system enters chaos in terms of a few bifurcations.

$$
\lim _{n \rightarrow \infty} \gamma_{n}=\gamma_{\infty}=0.164729 \text {. }
$$

Table 1 Parameters of mapping eq. (5) at the bifurcation points

\begin{tabular}{ccc}
\hline Bifurcation case & Bifurcation value $\gamma_{n}$ & $\begin{array}{c}\text { Difference ratio } \\
\left(\gamma_{n}-\gamma_{n-1}\right) /\left(\gamma_{n+1}-\gamma_{n}\right)\end{array}$ \\
\hline $1 \rightarrow 2$ & 0.1396 & \\
$2 \rightarrow 4$ & 0.1594 & 4.6817 \\
$4 \rightarrow 8$ & 0.1637 & 4.6457 \\
$8 \rightarrow 16$ & 0.1646 & 4.6547 \\
$\vdots$ & $\vdots$ & $\vdots$ \\
Bifurcation $\rightarrow$ chaos & 0.1647 & 4.669201661 \\
\hline
\end{tabular}

(iii) The self-similar fractal of the final-state diagram. As parameter $\gamma$ increases, there is an already well-defined path that moves from one state, the ordered state, into another state, the chaotic state. Moreover, the path resulting from the analysis is universal.

However, the structure of this universal path is a selfsimilar fractal. We now investigate a close-up of Figure 2. From one close-up to another, it is seen that the series of distances is not strictly a geometric series, as the scale factor changes slightly, approaching $\delta=4.669 \cdots$ in the horizontal direction and $\alpha=2.502 \cdots$ in the vertical direction [25].

Figure 4 shows the sequence of close-ups. This figure resembles the entire structure if we skip the other branch. Moreover, by magnifying the rectangular area, and turning the result upside down, we obtain the second close-up. In the same way, the third close-up is obtained. We now notice that the vertical axes of the first and third magnifications are inverted, and the color of the images fades gradually because the number of sample points decrease. Theoretically,
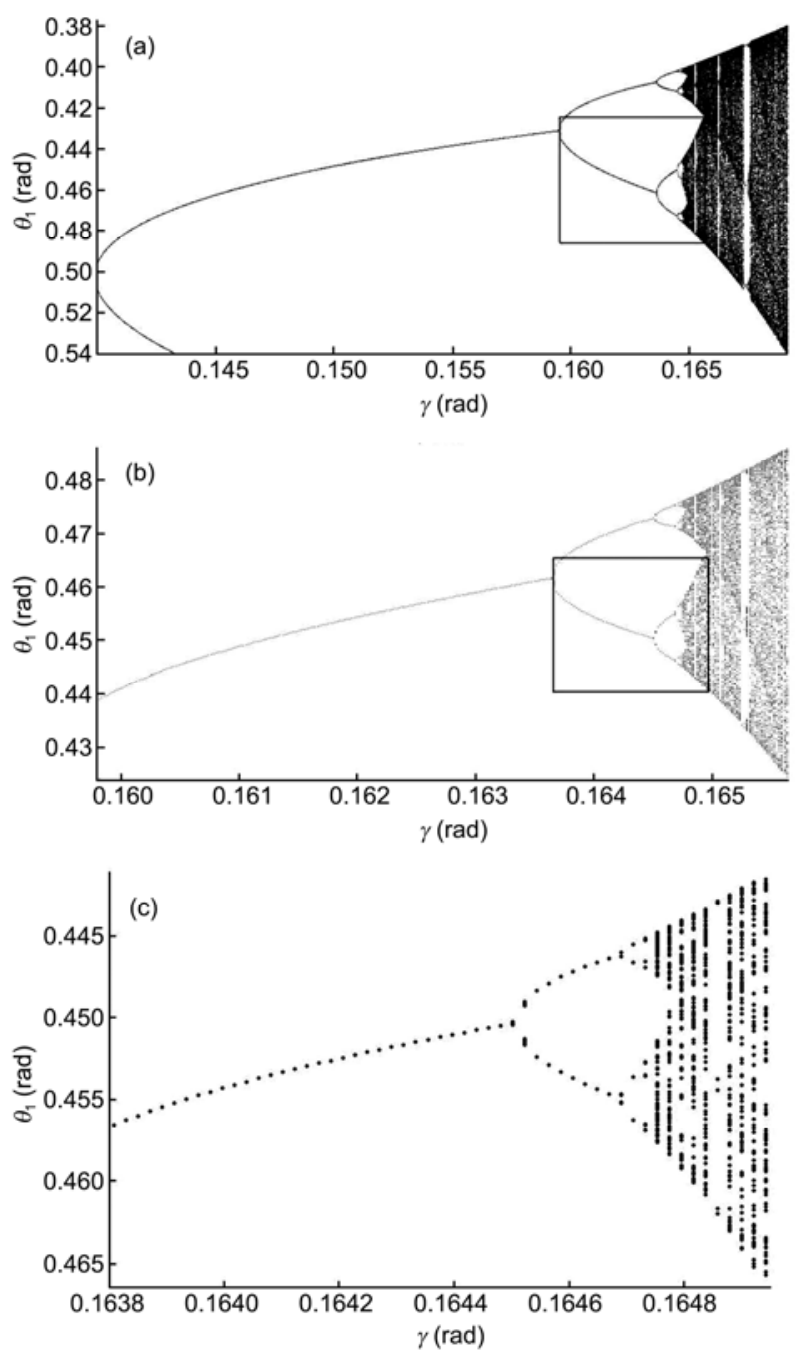

Figure 4 Self-similarity of Feigenbaum diagram. 
this can go on infinitely, requiring only more detailed areas and more iterations. Then the final-state diagram obtained is a geometric fractal, which has a structure similar to itself.

\subsection{The entrance of chaos}

Now to highlight the universality of the Feigenbaum constant $\delta$, there is a threshold 0.164729 , i.e., the Feigenbaum point $\gamma_{\infty}$, which causes the number of gait bifurcations of the robot to no longer increase. This indicates the end of the state of the period doubling bifurcation. The Feigenbaum point divides the final-state diagram into two entirely different parts: the first part is the period doubling tree, while the second part is the area of chaos. However, the second part is not the total chaotic area. The complicated structure is discussed step by step below.

(i) Rule of the final-state of the period doubling bifurcation. The period doubling bifurcation system has an approximately self-similar fractal. The self-similarity of the period doubling bifurcation tree from $\gamma=0.13$ to $\gamma_{\infty}$ is contained in the self-similarity features of the final-state diagram (shown in Figure 2). However, branches of the tree are not exact copies of the tree. Here, "self-similarity" is used to present intuitiveness instead of precision.

In Figure 5, the period doubling tree is still obtained using the scale factors $\delta$ and $\alpha$. Here the difference in the size of each branch is overlooked, and the set consisting of all leaves of the bifurcation tree, the Cantor set, is focused on. Addressing for branches and leaves of the bifurcation tree is introduced. Once bifurcation occurs, the branch at a lower place is marked as "L" (low) and the one at a higher place as " $\mathrm{H}$ " (high). When two branches bifurcate four branches, the upper two are labeled HH and HL, respectively, while the lower two are labeled LH and LL, respectively. This is the 2nd stage of the hierarchical addressing. Similarly, branches in the 3rd stage are labeled HHH, HHL, HLH, HLL, LHH, LHL, LLH, and LLL as shown in Figure 5. Then, for the $k$-th stage, $2^{k}$ sub-branches are annotated with $k$ letters. The leaves have address strings of infinite length. The first $k$

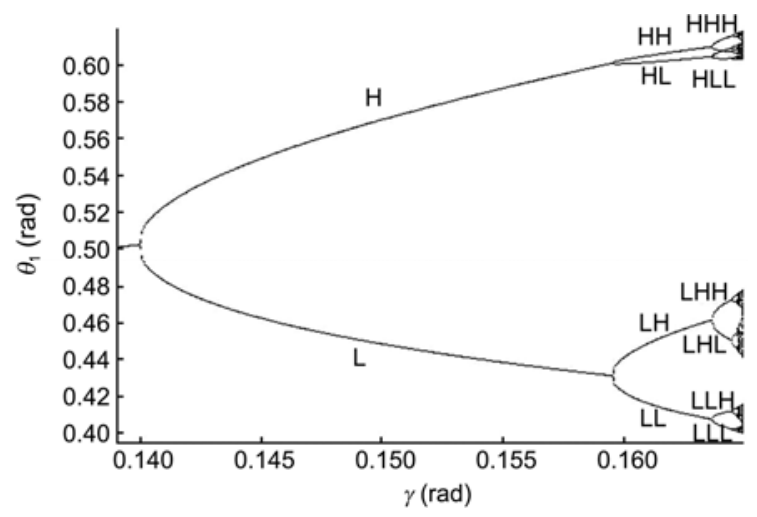

Figure 5 Address label of each branch of the period doubling tree. letters of each address indicate that a certain leaf belongs to the sub-branch of the $k$ th stage. Therefore, leaves of the tree create a self-similar Contor set.

(ii) Dynamic features of Feigenbaum points $\gamma_{\infty}$. For all values of $\gamma$ from $\gamma_{1}$ to $\gamma_{\infty}$, we observe steady periodic orbits, as in the final state. Now there is an unavoidable question: what dynamic features does the robot present when $\gamma_{1}=\gamma_{\infty}$ ? First, the dynamic features of the robot system at period doubling bifurcation are considered. The system has a steady periodic oscillation with a period of 8 when $\gamma \in\left(\gamma_{3}, \gamma_{4}\right)$. Three letters are needed for the address, with the address mapping:

$$
\begin{aligned}
& \mathrm{LLL} \rightarrow \mathrm{HLL} \rightarrow \mathrm{LHH} \rightarrow \mathrm{HHL} \rightarrow \mathrm{LLH} \\
& \rightarrow \mathrm{HLH} \rightarrow \mathrm{LHL} \rightarrow \mathrm{HHH} \rightarrow \text { LLL. }
\end{aligned}
$$

The order does not change no matter what the initial state is. In addition, there are the following rules in the mapping: (a) the address repeats once every 8 iterative processes, which corresponds to 8-periodic bifurcation; (b) for the first letter of each address, the iteration occurs as 2-periodic bifurcation $\mathrm{L} \rightarrow \mathrm{H} \rightarrow \mathrm{L}$; (c) for the first 2 letters of each address, the iteration occurs as 4-periodic bifurcation: $\mathrm{LL} \rightarrow \mathrm{HL} \rightarrow \mathrm{LH} \rightarrow \mathrm{HH} \rightarrow \mathrm{LL}$; (d) the address at the top of the diagram, $\mathrm{HHH}$, must be followed by the one at the bottom, i.e., LLL. It can easily be seen that the above 4 rules determine the entire sequence, that is, an 8-periodic walking gait of the biped robot system. Meanwhile, this has definite significance in physics.

There is a delicate balance in the energy conversion in a steady periodic gait of the robot, which causes the adjustment of the gait when parameters of the model change. When $\gamma<\gamma_{1}$, the energy provided by gravity is exactly consumed at the collision in the motion of each step. Thus the gait of the left leg is the same as that of the right leg, which is represented as a single-periodic walking gait.

When $\gamma_{1}<\gamma<\gamma_{2}$, the energy lost at the collision of the foot and the floor is not enough to consume the energy provided by gravity, which requires a great adjustment in the gait. The gaits of both legs are no longer consistent; the length of one step is longer, and the other shorter, i.e., by way of 2-periodic bifurcation $\mathrm{L} \rightarrow \mathrm{H} \rightarrow \mathrm{L}$. Thus the energy in the process of two steps will balance. The phenomenon above can also explain why a limp is more laborious in real life. When $\gamma_{2}<\gamma<\gamma_{3}$, the energy balance is barely achieved relying only on the different gaits between the two legs during two successive steps. Different gaits are needed between two successive steps of each leg to consume the additional energy caused by the increase in $\gamma$, and thus, the gait of the robot resembles $\mathrm{LL} \rightarrow \mathrm{HL} \rightarrow \mathrm{LH} \rightarrow \mathrm{HH} \rightarrow \mathrm{LL}$. Here we refer to the different gaits between two successive steps of the left leg as: left-first, left-second, and gaits of the right leg as: right-first, right-second. These correspond to the labels HH, HL, LH and LL, respectively. When $\gamma_{3}<\gamma<\gamma_{4}$, a 
further adjustment in the gait is necessary to perform the energy balance. The motion of the robot repeats once every 8 steps, as described in rule (a).

Now, we can clarify the address mapping rules for 8-periodic gait. In rule (b), the first letter $\mathrm{H}$ or $\mathrm{L}$ of the address corresponds to the left, right leg of the robot, respectively. In rule (c), for HH, HL, LH and LL, the first letter has the same meaning as in rule (b), and the second letter $\mathrm{H}$ or L corresponds to the front step or the back step. In rule (d), after HHH there must be LLL, because firstly, in the first two letters, LL comes right after HH; and secondly, when the gait is $\mathrm{HHH}$, the energy lost at the collision is at its maximum, and is far greater than the force of gravity, which causes the kinetic energy to be at its the lowest, and the speed of motion to be the slowest, and thus the steplength is reduced to the minimum, LLL.

The same reasoning allows us to determine the address sequence described by $2^{k}$-periodic orbit $(k=4,5, \ldots)$. Obviously, $2^{k}$ gaits are deduced from $2^{k-1}$ gaits. However, at each periodic bifurcation, the dynamic features of the iteration become much more complicated, although they share the same mechanism. The final state of the iterative system is an infinite aperiodic trajectory as $k \rightarrow \infty, \gamma=\gamma_{\infty}$. Each address has a one-to-one mapping with each point in the Cantor set. This can be considered to be the first mark of chaos, through which the robot gait enters chaos.

\subsection{Chaos mirror}

For the second part of the final-state diagram, the parameter is between the Feigenbaum point $\gamma_{\infty}$ and $\gamma=\gamma_{\text {end }}=0.172677$ (as shown in Figure 6). At a generalized level, this part of the diagram is usually called 'the chaos mirror of the period doubling bifurcation tree'. In fact, though in a chaotic area, it still has periodicity in reverse order. Meanwhile, in the place where chaos dominates, the gait sequence becomes infinitely complicated. We can accurately predict the dynamic features of the first part of the final-state diagram for each value of $\gamma$; whereas it is quite difficult for us even to differentiate the steady period from the features of chaos.

(i) Abruption of the sub-band. On the right-side of the

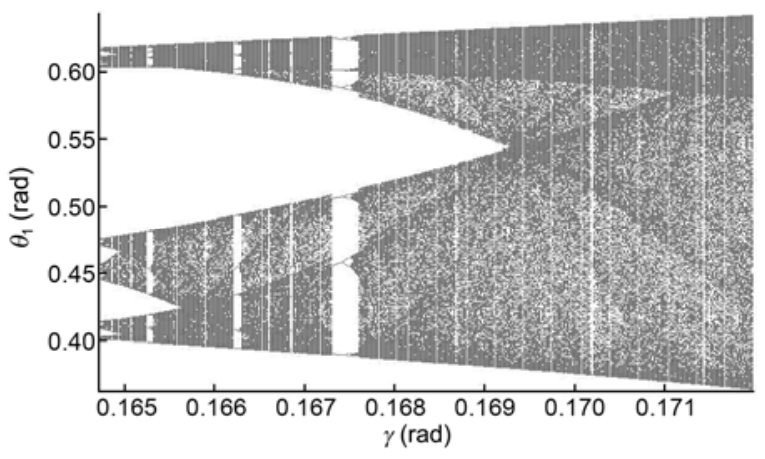

Figure 6 Chaos area of the final-state diagram. final-state diagram, i.e., when $\gamma=\gamma_{\text {end }}$ the graph of $S_{\gamma}(\boldsymbol{v})$ crosses the area $[0.35,0.63]$, the chaos sequence of the robot gait scatters throughout the entire vertical region. For any value of the parameter in the final-state diagram, it appears that there is a band structure resulting from the uneven distribution of points in the vertical direction. Points appear to converge on some lines, and each is the border of a sub-band, which is encapsulated with dynamic features of chaos. For $\gamma_{\text {end }}$, there is only one sub-band crossing the whole area of [0.35, 0.63]. As $\gamma$ decreases, the width of the sub-band becomes small. When $\gamma=m_{1}$, the sub-band splits into two parts; and when $\gamma=m_{2}$, it splits again.

(ii) Merging points. By magnifying the rectangular area in Figure 7(a) with the parameter between $\gamma_{\infty}$ and $m_{1}$, we obtain Figure 7(b), which shows more points where subbands are abrupt. In fact, at the places where the $2 \mathrm{nd}$, 4th, 8 th, $\cdots, 2^{k}$ th, sub-bands occur from one band, there is an infinite sequence with the parameter values in reverse order $m_{1}$, $m_{2}, m_{3}, \cdots$. Given this, it is amazing that the sequence converges accurately to the Feigenbaum point, i.e., $m_{\infty}=\gamma_{\infty}$. This is further evidence that the final-state diagram has self-similarity at the Feigenbaum point. Moreover, the distance of adjacent merging points in one sub-band, $d_{k}=a_{k+1}-a_{k}$, obeys the rule of period doubling bifurcation. When $k \rightarrow \infty$, the ratio $d_{k} / d_{k+1}$ converges to the Feigenbaum constant $\delta=$ $4.669 \cdots$. That is why the second part of the final-state diagram is called "the chaos mirror of the period doubling tree".
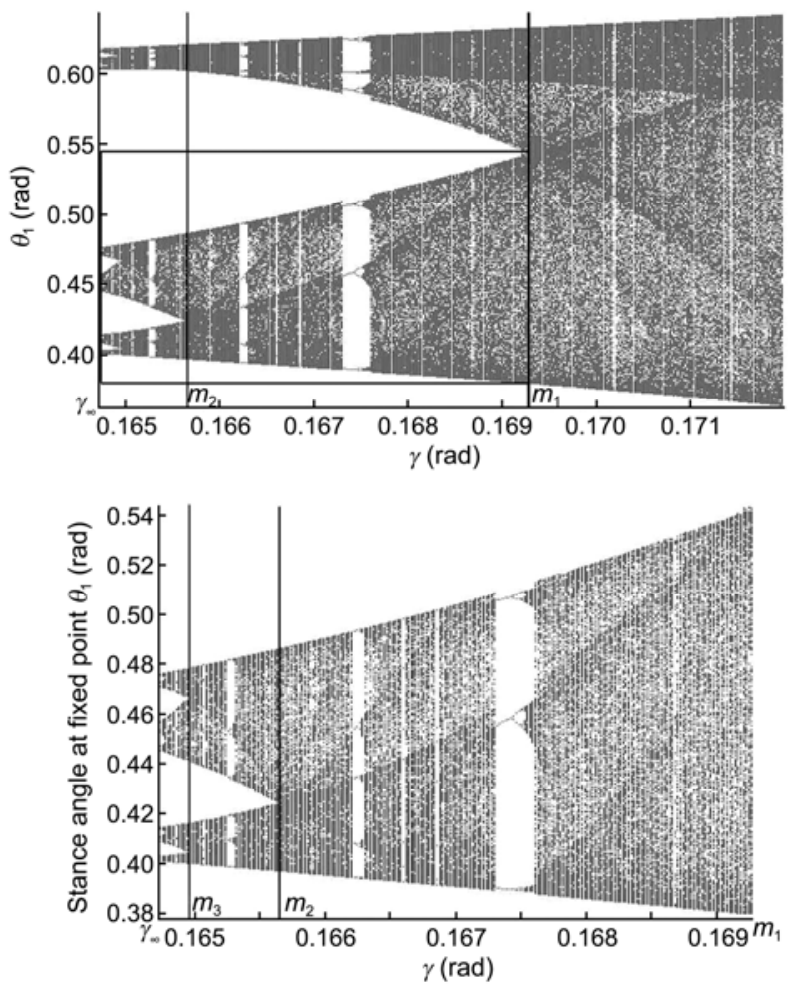

Figure 7 Abruption of the sub-band (a) abruption of the sub-band in the final-state diagram; (b) further abruption of the sub-band. 
To obtain more clarity, the generalized symmetry of sub-bands and the period doubling tree are analyzed in detail. The curve $S_{\gamma}$ in Figure 8(a) shows a typical gait iteration sequence; when $\gamma=0.169$, the value of $\gamma$ is a little lower than $m_{1}$. The behavior of dynamic features is chaotic, but it oscillates between two definitely different sub-bands step by step, that is, it switches between both legs of the robot. In other words, when we observe the dynamic features of $S_{\gamma}^{2}$, we find that the iteration points move chaotically, either just in the upper sub-band or in the lower sub-band, which is shown as two curves of $S_{\gamma}^{2}$ in Figure 8(a). In the same way, access times of the iteration orbit of the gait to different areas of the sub-band are calculated in Figure 8(b). The regions of both sub-bands are clear, which corresponds to the motion area of the left and right legs. In conclusion, the abruption of the first sub-band is essentially a period doubling bifurcation.

For the robot in the area after $\gamma<m_{1}$, the chaotic gait area of both legs transforms into 2 separate areas instead of being connected. Similarly, after $\gamma<m_{k}$, the chaotic gait area transforms into $2^{k}$ areas. So the essence of the abruption of the sub-band is period doubling bifurcation, and is thus also the mirror of the period doubling tree.

(iii) Rule for the final-sate of the period doubling bifurcation. In the section of the period doubling bifurcation,
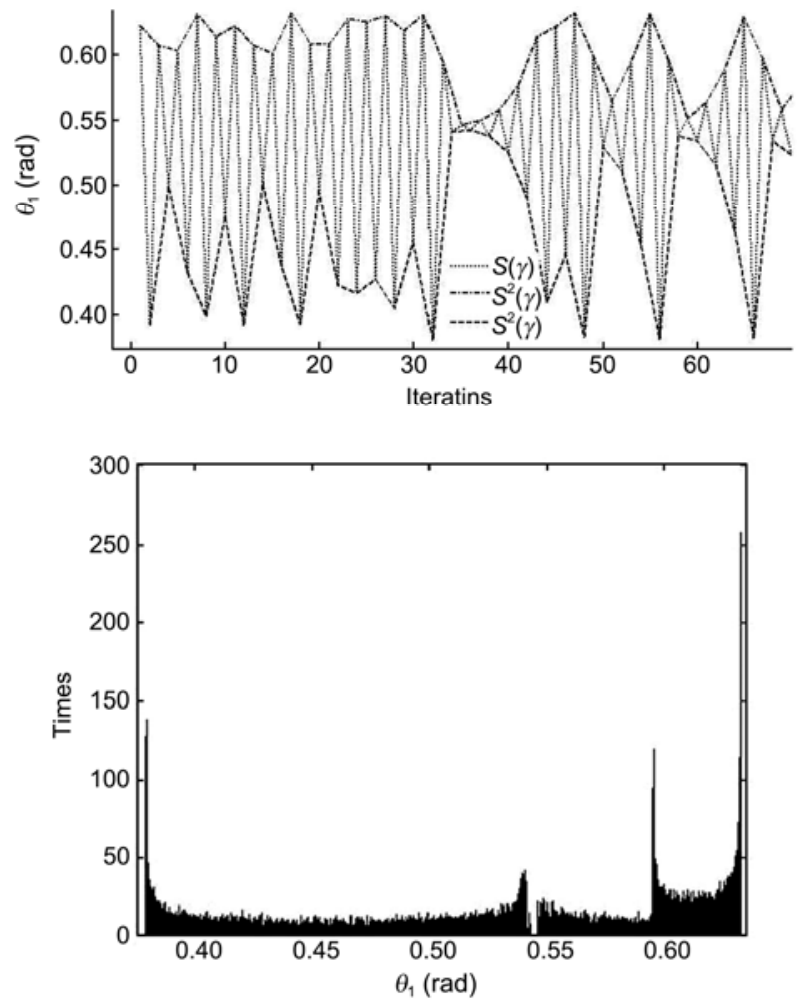

Figure 8 Abruption of sub-bands (a) two disconnected sub-bands of each leg when $\gamma$ is a little smaller than $m_{1}$; (b) access times of different areas of sub-bands. there is a periodic rule in the address order that LLL follows immediately after $\mathrm{HHH}$. This raises the next problem, whether the rule works in the second part of the final-state diagram. According to Figure 9, which shows the three iterative processes (marked as 2, 3, 4) of the maximum at value $\gamma_{\text {end }}$, the periodic rule seems to work. Now, here is another assumption: if the rule also applies to the chaotic gait, then in the whole parameter region of the final-state diagram, critical curves of sub-bands with a period of 8 will be obtained, if we compute the first 7 iterative processes $S_{\gamma}^{7}\left(\boldsymbol{v}_{\max }\right)$ of each maximum $\boldsymbol{v}_{\max }$ corresponding to values of $\gamma$, which are depicted as the bright concentrated line in Figures 6 and 7.

Now we calculate $v_{\max }$ and the first 7 iterative processes. All the 8 iterative processes are shown in Figure 10 (i.e., from $\boldsymbol{v}_{\max }$ to $\left.S_{\gamma}^{7}\left(\boldsymbol{v}_{\max }\right)\right)$. In the area of the period doubling bifurcation, the curves totally overlap the 8-periodic gait sequence. In the chaotic area, these curves obviously correspond to the main bands, which are depicted as bright parts in the final-state diagram. It is obvious that there is a more exact subtle relationship among sub-band structures of the final-state diagram when the times of the iterative processes increase; however, the gait sequence loses periodicity bit by bit as we proceed with the iterative process. Hence, although

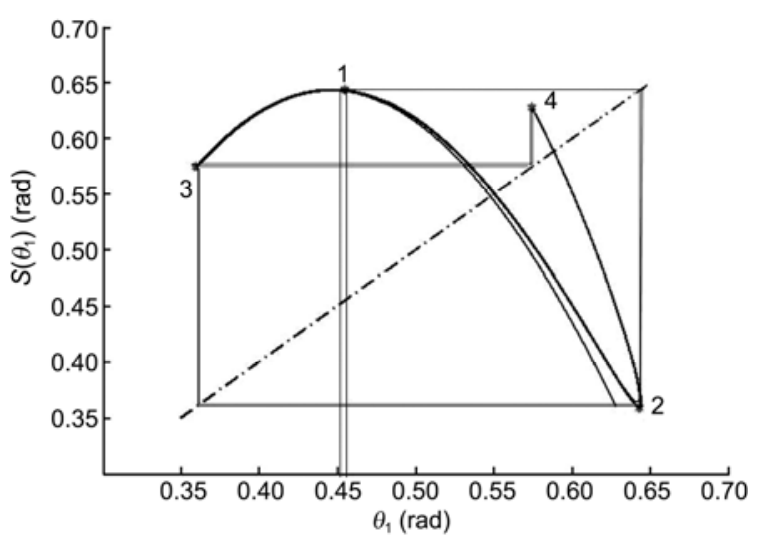

Figure 9 Three iterative processes, beginning with the area that is close to the maximum value.

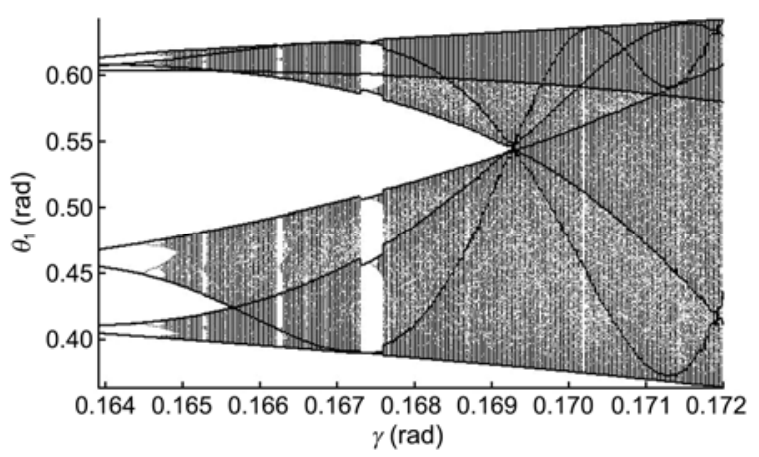

Figure 10 Critical curves of sub-bands. 
the chaotic gait of the robot appears to be quite disordered, it is highly ordered at a deeper level, and shows a refined structure at some stage.

\section{Conclusion}

This paper focused on a constructed biped passive homogeneous compass-gait model. The intrinsic features whereby a change in parameters leads to a change in the gait, were analyzed, revealing the inherent laws of the phenomenon of the period doubling bifurcation and chaos. Simulation of the increase in parameter $\gamma$ indicated that the way the biped passive dynamic walking robot gait enters chaos is by period doubling bifurcation. Moreover, when $\gamma$ is between 0.1 and 0.172677 , the final-state diagram of the robot system has the geometrical features of a self-similar fractal.

When period doubling bifurcation occurs, the robot has a definite and regular gait sequence to ensure its energy is balanced. The relationship among bifurcation points suggests that when the gait moves from bifurcation into chaos, the process obeys the law that they have the same Feigenbaum universal constant $\delta$. Moreover, this law is also shared by all the period doubling bifurcation phenomena.

At the start of chaos, the Feigenbaum point, the final-gait sequence is given by an infinite-length aperiodic trajectory in the Cantor set, which infinitely approaches each point in the Cantor set. In the chaotic stage, sub-bands of the finalstate diagram present periodic doubling bifurcation numbers in reverse order, and also have a convergence speed of $\delta$. Moreover, the critical curves of the sub-band present a more exact subtle relationship among the sub-band structures, and determine the change rule for the motion area of each leg.

This work was supported by the National Natural Science Foundation of China (60905049) and the self-managed Project of State Key Laboratory of Robotic Technology and System in Harbin Institute of Technology (200804C).

1 McGeer T. Passive dynamic walking. Int J Robot Res, 1990, 9: 62-82

2 Wisse M, Hobbelen D G E, Schwab A L. Adding an upper body to passive dynamic walking robots by means of a bisecting hip mechanism. IEEE T Robot, 2007, 23: 112-123

3 Collins S, Ruina A, Tedrake R, et al. Efficient bipedal robots based on passive-dynamic walkers. Science, 2005, 307: 1082-1085

4 Collins S H, Ruina A. A bipedal walking robot with efficient and human-like gait. In: 2005 IEEE Int Conf Robot Autom, 2005 Apr 18-22, Barcelona, Spain. IEEE Press, 2005. 1983-1988

5 Huang Y, Wang Q N, Xie G M, et al. Optimal mass distribution for a passive dynamic biped with upper body considering speed, efficiency and stability. In: 2008 8th IEEE-RAS Int Conf on Humanoid Robots, 2008 Dec 1-3, Daejeon, Korea. IEEE Press, 2008. 515-520

6 Wisse M. Essentials of dynamic walking: Analysis and design of two-legged robots. Doctorial Dissertation. Netherlands: Delft University of Technology, 2004

7 Piiroinen P, Dankowicz H. Low-cost control of repetitive gait in passive bipedal walkers. Int J Bifurcat Chaos, 2005, 15: 1959-1973

8 Hiskens I A. Stability of hybrid system limit cycles: Application to the compass gait biped robot. In: Proceedings of the 40th IEEE Conf on Decision and Control, 2001 Dec 4, Orlando, America. IEEE Press, 2001. 774-779

9 Hiskens I, Pai M. Trajectory sensitivity analysis of hybrid systems. IEEE Trans Circuits I, 2000, 47: 204-220

10 Hansen A, Childress D, Miff S, et al. The human ankle during walking: Implications for design of biomimetic ankle prostheses. J Biomech, 2004, 37: 1467-1474

11 Ramamoorthy S, Kuipers B J. Trajectory generation for dynamic bipedal walking through qualitative model based manifold learning. In: IEEE Int Conf on Robotics and Automation, 2008 May 19-23, Pasadena California, America. IEEE Press, 2008. 359-366

12 Winte D. Biomechanics and Motor Control of Human Movement. Hoboken, NJ: John Wiley and Sons, 1990

13 Liu N, Li J F, Wang T S. The effects of parameter variation on the basins of attraction of passive walking models. In: IEEE Int Conf on Mechatronics and Automation, 2007 Aug 5-8, Harbin, China. IEEE Press, 2007. 1908-1913

14 Asano F, Luo Z W. On energy-efficient and high-speed dynamic biped locomotion with semicircular feet. In: IEEE/RSJ Int Conf on Intelligent Robots and Systems, 2006 Oct 9-15, Beijing, China. IEEE Press, 2006. 5901-5906

15 Asano F, Luo Z W. On efficiency and optimality of asymmetric dynamic bipedal gait. In: 2009 IEEE Int Conf on Robotics and Automation, 2009 May 12-17, Kobe, Japan. IEEE Press, 2009. 1972-1977.

16 Garcia M, Chatterjee A, Ruina A. Efficiency, speed, and scaling of two-dimensional passive-dynamic walking. Dynam Stabil Syst, 2000, 15: 75-99

17 Goswami A, Thuilot B, Espiau B. Compass-like biped robot part I: Stability and bifurcation of passive gaits. Institut National de Recherche en Informatique et en Automatique, 1996, 2996: 1-86

18 Garcia M, Chatterjee A, Ruina A, et al. The simplest walking model: Stability, complexity, and scaling. J Biomech Eng, 1998, 120: 281-288

19 Asano F, Luo Z W. Pseudo virtual passive dynamic walking and effect of upper body as counterweight. In: 2008 IEEE/RSJ Int Conf on Intelligent Robots and Systems, 2008 Sep 22-26, Nice, France. IEEE Press, 2008. 2934-2939

20 Liu Z, Tian Y T, Zhang P J, et al. The analysis on bifurcation and chaos in the compass-gait biped. In: 2007 IEEE Int Conf on Robotics and Biometrics, 2007 Dec 15-18, Shenyang, China. IEEE Press, 2007. 972-977

21 Spong M W, Bullo F. Controlled symmetries and passive walking. IEEE Trans Automat Contr, 2005, 50: 1025-1031

22 Bullo F, Zefran M. Modeling and controllability for a class of hybrid mechanical systems. IEEE Trans Robot Autom, 2002, 18: 563-573

23 Zhang J, Zhao M G, Dong H. Effect of energy feedbacks on virtual slope walking: I. Complementary energy feedback. In: 2009 IEEE Int Conf on Robotics and Automation, 2009 May 12-17, Kobe, Japan. IEEE Press, 2009. 1959-1965

24 Bu S, Wang B H, Jiang P Q. Detecting unstable periodic orbits in chaotic systems by using an efficient algorithm. Chaos Soliton Fract, 2004, 22: 237-241

25 Peitgen H O, Jurgens H, Saupe D. Chaos and Fractals: New Frontiers in Science. 2nd ed. New York: Springer-Verlag, 2004

26 Gao Q Y, Zhang L, Zhang X, et al. Quasi-periodicity in the autonomous glycolytic system. Chin Sci Bull, 2005, 50: 1839-1842

27 Zheng S Y, Guo H X, Li Y A, et al. A new method for detecting line spectrum of ship-radiated noise using Duffing oscillator. Chin Sci Bull, 2007, 52: 1906-1912

Open Access This article is distributed under the terms of the Creative Commons Attribution License which permits any use, distribution, and reproduction in any medium, provided the original author(s) and source are credited. 